\title{
Coupling of flexural and longitudinal damped vibration in a two-layered beam
}

\author{
F. Pourroy ${ }^{\mathrm{a}}$, S. Shakhesi ${ }^{\mathrm{a}}$ and P. Trompette ${ }^{\mathrm{b}}$ \\ ${ }^{a}$ Laboratoire des Sols, Solides et Structures, BP53, \\ 38041 Grenoble Cedex 9, France \\ ${ }^{\mathrm{b}}$ Institut des Sciences et Techniques de l'Ingénieur de \\ Lyon, 69000 Lyon, France
}

Received 18 November 1996

Revised 6 November 1998

In dynamics, the effect of varying the constitutive materials' thickness of a two-layered beam is investigated. Resonance frequencies and damping variations are determined. It is shown that for specific thicknesses the coupling of longitudinal and flexural vibrations influences the global modal damping ratio significantly.

Keywords: Dynamics, coupling, damping, two-layered beam

\section{Introduction}

Many papers have been devoted to the analysis of the dynamical behavior of multilayered structures, specially plates and beams. Different hypotheses on the displacement fields in each layer have been formulated: linear with or without shear (see for example [3, $4,6,10,14]$ ) or non linear (see for example $[1,2,5,9,13$, 18]). They lead to more or less sophisticated equivalent homogeneous beam or plate models (analytical or finite element ones), or to multilayered models in which each layer behavior is defined from its own displacement field.

Some papers more particularly concern the influence of coupling effects on the analysis of multilayered structures. Sivadas and Ganesan [16] analyzed effects of coupling on natural frequencies of circular cylindrical shells (coupling between symmetric and antisymmetrical modes). Mead and Markus [11] studied wave motion in a three layer beam and discussed a coupling phenomenon between longitudinal and flexural waves. Owen and $\mathrm{Li}$ [12] computed natural vibrations of laminated anisotropic simply supported plates and emphasized the influence of the coupling between bending
Table 1

Mechanical properties of constitutive materials

\begin{tabular}{cccccc}
\hline & $E(\mathrm{MPa})$ & $\nu$ & $\rho\left(\mathrm{kg} / \mathrm{m}^{3}\right)$ & $\eta_{\mathrm{n}}(\%)$ & $\eta_{\mathrm{s}}(\%)$ \\
\hline Aluminium & 70000 & 0.3 & 2700 & 0.1 & 0.1 \\
Polyester & 3450 & 0.41 & 1220 & 1.4 & 2.2 \\
\hline
\end{tabular}

and stretching on both global and local results. Sun and Chin [17] showed that bending-extension coupling in unsymmetrical cross-ply laminates can produce large deflection effect even in the small deflection range. Hwang and Gibson made a review of application of strain energy-based finite element techniques in the analysis of composite damped structures [7]. At the same time, they presented a strain energy-based approach for studying the effects of vibration coupling on the damping of symmetric composite laminated cantilever beams [8]. Shearing-stretching and bendingtwisting effects were considered.

Recently a comparison of the results given by some of the different multi-layered beam flexural vibration models has been undertaken by Shakhesi [15]. The influence of an accurate representation of the shear in the different layers has been outlined. On investigating the evolution of the resonance frequencies and their corresponding damping loss factors of a two-layered beam when the thicknesses of the two constitutive materials vary, a surprising coupling effect between flexural and longitudinal waves has been observed and is presented here.

\section{The two-layered beam}

The two-layered cantilever beam of interest has the following dimensions: it is $300 \mathrm{~mm}$ long and $30 \mathrm{~mm}$ wide. Total thickness is $40 \mathrm{~mm}$ and the characteristics of the constitutive materials are listed in Table 1. Assumption is made that these materials are both homogeneous and isotropic. Hence, material damping is characterized by the material loss factor in tensioncompression $\eta_{\mathrm{n}}$ and the material loss factor in shear $\eta_{\mathrm{s}}$ with: 


$$
\eta_{\mathrm{n}}=\frac{E^{\prime}}{E}, \quad \eta_{\mathrm{s}}=\frac{G^{\prime}}{G}
$$

where $E, E^{\prime}$ and $G, G^{\prime}$ are real and imaginary parts of complex Young and shear moduli.

Some beam configurations have been considered with variations in each layer's thickness ranging from 0 to $40 \mathrm{~mm}$, while keeping the beam total thickness constant.

Since the study consists in an unsymmetrical composite beam in flexural vibration the coupling phenomenon of interest is the interaction between bending and extension.

\section{Finite element models}

Two different models are considered, both using a higher order shear deformation theory (HSDT):

- Model I, in which coupling effects are neglected, is based on the following displacement field:

$$
\begin{aligned}
& u(x, z, t)=z \theta(x, t)+z^{3} \zeta(x, t), \\
& w(x, t)=w_{0}(x, t) .
\end{aligned}
$$

- Model II includes coupling effects and is based on the following displacement field:

$$
\begin{aligned}
& u(x, z, t)=u_{0}(x, t)+z \theta(x, t)+z^{3} \zeta(x, t), \\
& w(x, t)=w_{0}(x, t)
\end{aligned}
$$

where $(x, z)$ is the plane of flexion of the beam, $(u, w)$ are the displacements of a generic point $(x, z)$ in the beam at time $t$ and $\left(u_{0}, w_{0}\right)$ are the displacements of a point on the neutral axis of the beam. $\theta(x, t)$ is the rotation of the normal to the neutral axis and $\zeta(x, t)$ is the warping function of the section. The associated longitudinal and transverse shear strains can be calculated from Eqs (2) or (3) and the corresponding stresses can be expressed writing the constitutive equations for each layer. Using these expressions of strains and stresses, the principle of virtual work at each instant of time is written in terms of displacements, rotations and warping variables. According to the general finite element procedure, the beam is discretized into $N$ three-node elements with four degrees of freedom per node $\left(u_{0}, w_{0}, \theta\right.$ and $\left.\zeta\right)$ and the vector of displacements in each element $e$ is written in the following matrix form:

$$
\{u\}_{e}=[N]_{e}\{\bar{u}\}_{e},
$$

where $[N]_{e}$ is the matrix of shape functions and $\{\bar{u}\}_{e}$ denotes the nodal displacement vector associated with element $e$.

The damping model used in the outcoming elements is based on the concept of specific damping capacity (SDC) which states:

$$
\psi_{i}=\frac{\Delta U_{i}}{U_{i}}
$$

where $\Delta U_{i}$ is defined as the energy absorbed by the structure and $U_{i}$ is the maximum strain energy per cycle of loading, for mode $i$ :

$$
\begin{aligned}
\Delta U_{i} & =\frac{1}{2}\left\{\phi_{i}\right\}^{\mathrm{T}}\left[K^{\prime}\right]\left\{\phi_{i}\right\}, \\
u_{i} & =\frac{1}{2}\left\{\phi_{i}\right\}^{\mathrm{T}}[K]\left\{\phi_{i}\right\} .
\end{aligned}
$$

$\left\{\phi_{i}\right\}$ is the $i$-th modal shape of the system, $[K]$ is the stiffness matrix and $\left[K^{\prime}\right]$ the damping matrix ([K] and $\left[K^{\prime}\right]$ are real and imaginary parts of the complex stiffness matrix). Provided that the damping is low, the following relation can be written:

$$
\psi=2 \pi \eta
$$

\section{Numerical results and coupling effect}

The two-layered beam described previously has been successively modelized using twenty finite elements derived from model I and model II. Natural frequencies and corresponding damping loss factors have been computed for the first two modes which are flexural ones. Figure 1 shows the effect of thickness distribution on the natural frequencies for modes 3 and 4. The curves concerning model II clearly exhibit two particular points where natural frequencies associated with modes 3 and 4 are very close. These two points correspond approximately to a $11 \mathrm{~mm}$ and a $33 \mathrm{~mm}$ polyester thickness (or $29 \mathrm{~mm}$ and $7 \mathrm{~mm}$ aluminium thickness).

Between these particular points, the evolution of the third natural frequency computed from model I very well fits that computed from model II. In return, when the polyester thickness is less than $11 \mathrm{~mm}$ or more than $33 \mathrm{~mm}$, model I gives results for mode 3 which are very close to those corresponding to mode 4 of model II.

The same phenomenon is observed on the curves of Fig. 2 which represent the variation of the modal damp- 


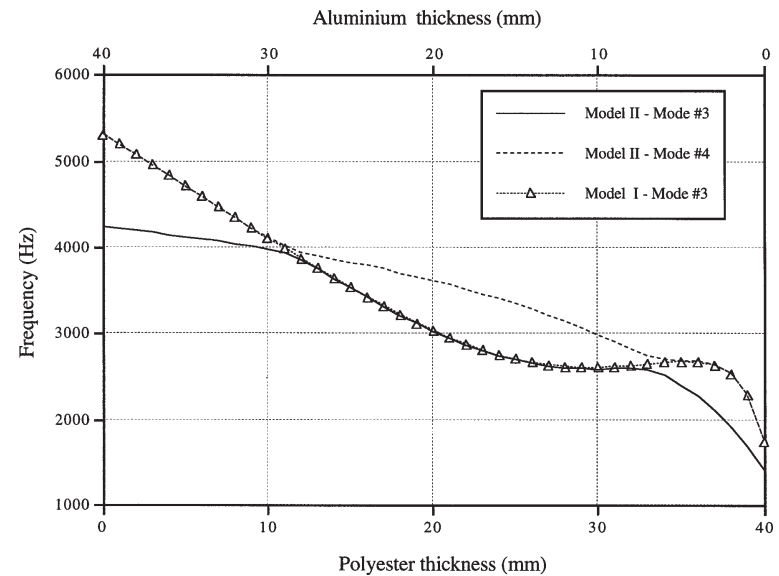

Fig. 1. The effect of thickness distribution on the natural frequencies.

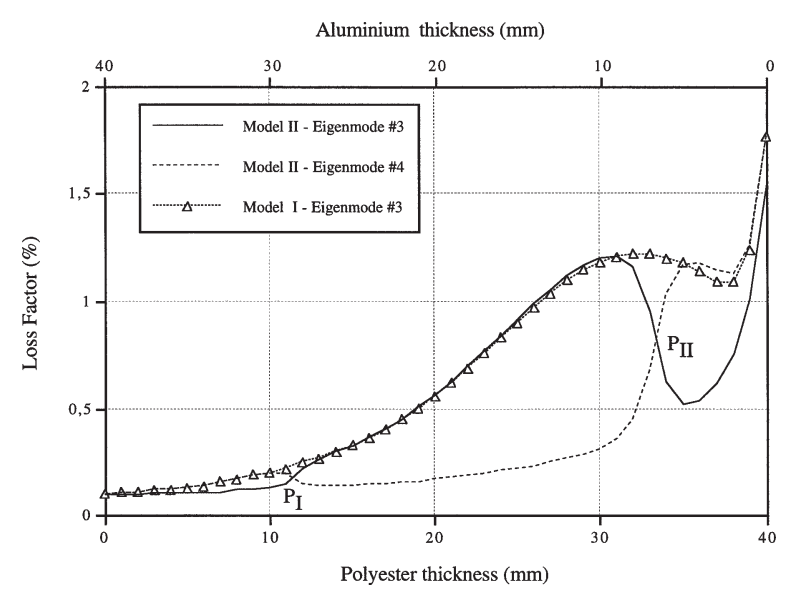

Fig. 2. The effect of thickness distribution on damping loss factor.

ing loss factors versus polyester thickness. The curve corresponding to model I-mode \#3 fits that of model II-mode \#3 or the one for model II-mode \#4 depending on thickness distribution between polyester and aluminium. Transition occurs at the same two particular points.

The third eigenmode derived from model I is clearly identified as a flexural mode of the beam. The third and fourth eigenmodes derived from model II can be identified from the decomposition of the total strain energy $U$ into a flexural strain energy $U_{\mathrm{f}}$ and a longitudinal strain energy $U_{1}$. In this way, the adimentional ratio $U_{\mathrm{f}} / U$ tends toward one for a flexural mode and the same ratio tends toward zero in the case of a longitudinal mode. The variation of $U_{\mathrm{f}} / U$ versus polyester thickness for the third and fourth eigenmodes derived from model II is shown on Fig. 3.

The resulting curves clearly show that in the range between $\mathrm{P}_{\mathrm{I}}$ and $\mathrm{P}_{\mathrm{II}}$ the third eigenmode derived from

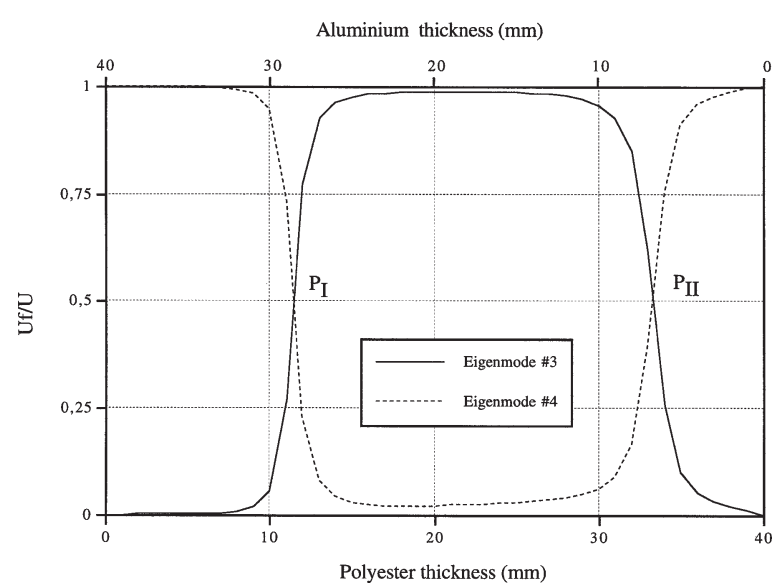

Fig. 3. The effect of thickness distribution on the modal strain energy (model II).

model II is a flexural one. On the other hand, when polyester thickness is below $\mathrm{P}_{\mathrm{I}}$ or above $\mathrm{P}_{\mathrm{II}}$, this third eigenmode corresponds with longitudinal vibrations. In that case, this is the fourth eigenmode which is a flexural one. This phenomenon gives an explanation of the observations made on the curves of Figs 1 and 2 and described below.

It is to be noticed that in the vicinity of the particular points $\mathrm{P}_{\mathrm{I}}$ or $\mathrm{P}_{\mathrm{II}}$ there is a coupling effect between both modes. The two points themselves correspond to the following partition of the strain energy:

$$
U=2 U_{\mathrm{f}}=2 U_{1}
$$

It can be seen from Fig. 3 that a slight coupling between longitudinal and flexural effects is remaining for all beam configurations in the range between $P_{I}$ and $\mathrm{P}_{\mathrm{II}}$.

The curves presented Fig. 2 showed the third and fourth mathematical eigenmodes. They can now be drawn again considering on the one hand the third mode where flexural energy is dominant and on the other hand the first mode governed by longitudinal energy. The new graph is presented Fig. 4. It can be seen from the curve that in the vicinity of the two points $P_{I}$ and $\mathrm{P}_{\mathrm{II}}$, the longitudinal damping loss factor rise to a maximum as the flexural damping loss factor fall to a minimum.

As has already previously seen with the strain energy, the total dissipated energy $\Delta U$ can be partitioned into a flexural dissipated energy $\Delta U_{\mathrm{f}}$ and a longitudinal dissipated energy $\Delta U_{1}$. The ratio $\Delta U_{\mathrm{f}} / \Delta U$ is used to represent the part of energy dissipated from flexural vibrations. Figure 5 shows the influence of thickness 


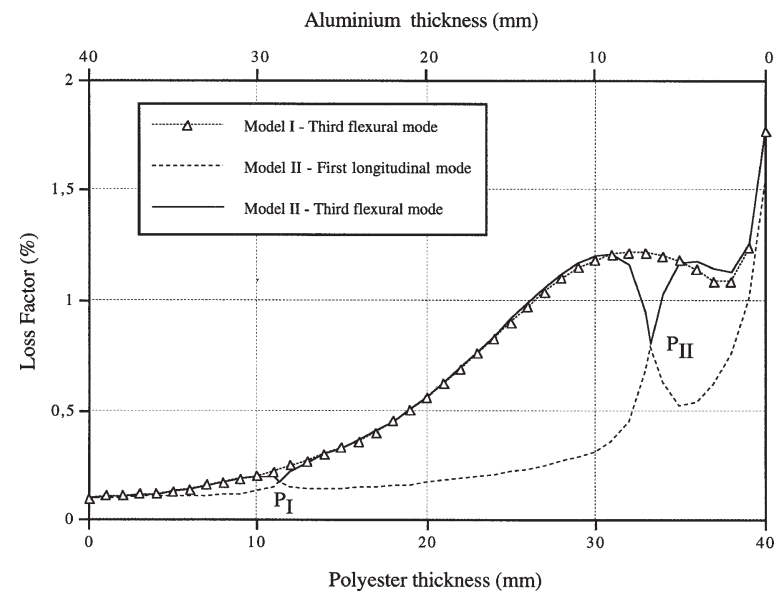

Fig. 4. Computations of damping loss factor for first longitudinal and third flexural mode.

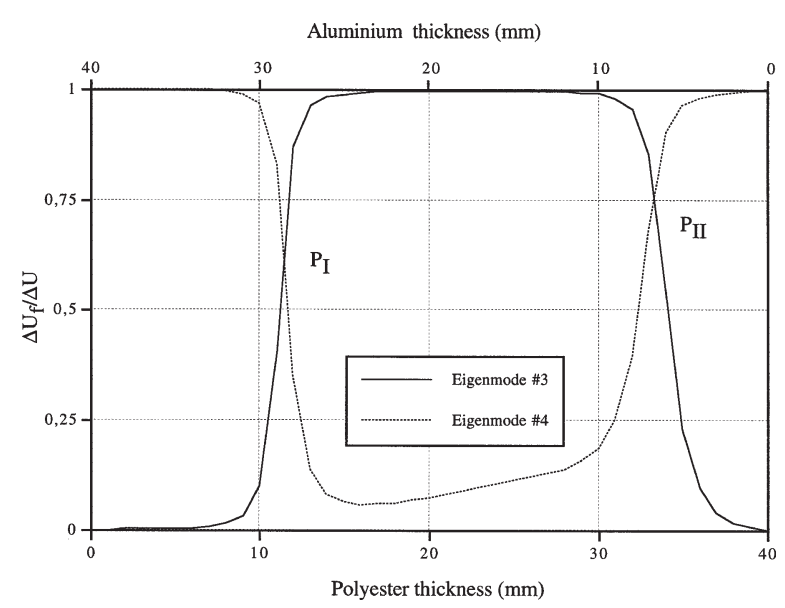

Fig. 5. The effect of thickness distribution on the dissipated energy (model II).

distribution on the dissipated energy ratio $\Delta U_{\mathrm{f}} / \Delta U$. It can be seen that for beam configurations $\mathrm{P}_{\mathrm{I}}$ and $\mathrm{P}_{\mathrm{II}}$ the energy is dissipated mainly by the way of flexural vibrations (i.e., flexural dissipated energy is about $75 \%$ of the total loss energy for the beam configuration at $\mathrm{P}_{\mathrm{II}}$ ). However, for the same beam configurations $\mathrm{P}_{\mathrm{I}}$ and $\mathrm{P}_{\mathrm{II}}$, it was shown from Fig. 3 that the total strain energy was equally distributed between longitudinal and flexural effects. This can be explained by the fact that flexural vibrations involve a damping loss factor $\eta_{\mathrm{s}}$ due to shear effects which is generally greater than the damping loss factor $\eta_{\mathrm{n}}$ which governs longitudinal vibrations. This is the case here with the polyester layer.

Another consequence is that between the two beam configurations at points $\mathrm{P}_{\mathrm{I}}$ and $\mathrm{P}_{\mathrm{II}}$, the third eigenmode, which is clearly ruled by the flexion, dissipates almost

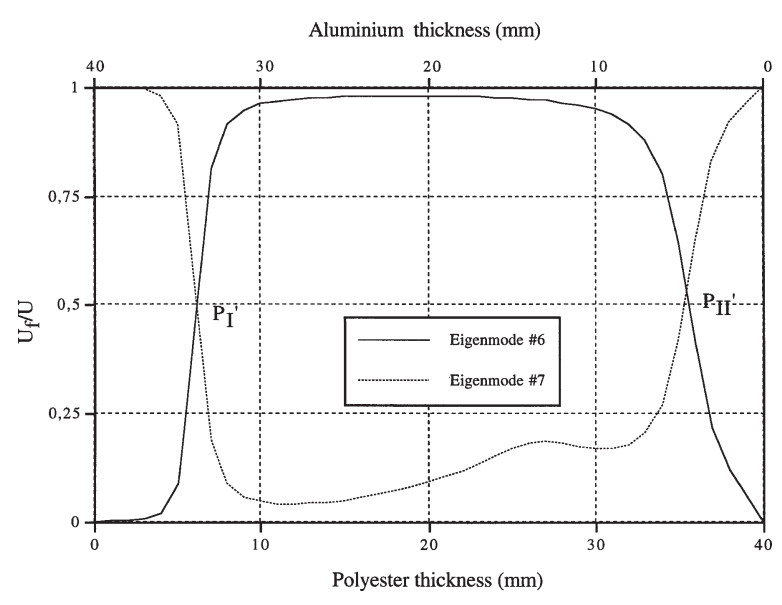

Fig. 6. The effect of thickness distribution on the strain energy (model II).

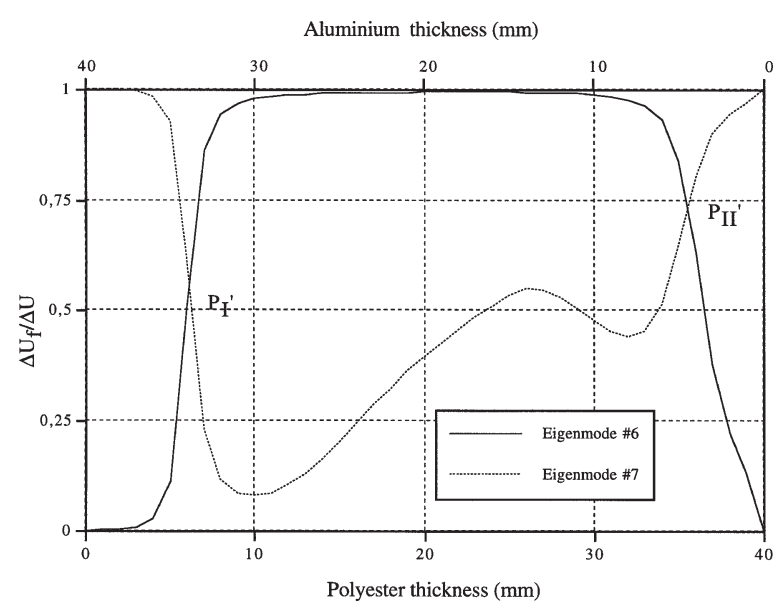

Fig. 7. The effect of thickness distribution on the dissipated energy (model II).

no longitudinal energy. On the other hand, although the fourth eigenmode is ruled by longitudinal vibrations, a significant part of loss energy is dissipated by the flexion. The corresponding curve shown by Fig. 5 gives a ratio $\Delta U_{\mathrm{f}} / \Delta U$ in the range from 0.1 to 0.2 , that is to say that from 10 to $20 \%$ of loss energy is dissipated by the flexion.

Similar coupling effects can be observed by studying of eigenmodes 6 and 7 for which the same conclusions can be drawn (see Figs 6 and 7 for the variation of $U_{\mathrm{f}} / U$ and $\left.\Delta U_{\mathrm{f}} / \Delta U\right)$. These eigenmodes alternatively correspond to the fifth flexural mode and the second longitudinal mode, depending on the distribution of thickness in the beam. One should notice the increasing influence of coupling effects in these higher 
modes and the shifting of the transition points from $\mathrm{P}_{\mathrm{I}}$ and $\mathrm{P}_{\mathrm{II}}$ to $\mathrm{P}_{\mathrm{I}}^{\prime}$ and $\mathrm{P}_{\mathrm{II}}^{\prime}$.

\section{Summary and conclusions}

The coupling effects of flexural and longitudinal vibrations have been pointed out from the damping and dynamic analysis of a two-layered beam. The effects of varying the constitutive materials' thickness of the beam have been investigated.

The results have shown that coupling effects occur more specially for some particular beam configurations (particular values of the thickness of the layers). A mechanical model including coupling capabilities will be very useful in such cases. Another practical aspect of these results is the possible use of the coupling phenomenon in the design of damping for vibration control (i.e., it is possible to increase the longitudinal damping capability of the beam by coupling longitudinal vibrations with highly damped flexural vibrations).

\section{References}

[1] G. Akhras, M.S. Cheung and W. Li, Finite strip analysis of anisotropic laminated composite plates using high-order shear deformation theory, Computers \& Structures 52(3) (1994), $471-477$.

[2] A.S. Bicos and G.S. Springer, Analysis of free damped vibration of laminated composite plates and shells, Int. J. Solids and Structures 25(2) (1989), 129-149.

[3] R.M. Crane, Analytical model for prediction of the damping loss factor of composite materials, Polymer Composites 13(3) (1992), 179-190.

[4] A. Fages and G. Verchery, Influence de la prise en compte du cisaillement transversal sur la calcul des fréquences propres des poutres sandwiches, J. de Mécanique Théorique et Appliquée 5(1) (1986), 73-93.

[5] A.K. Gosh and S.S. Dey, Free vibration of laminated composite plates - a simple finite element based on higher order theory, Computers \& Structures 52(3) (1994), 397-404.
[6] B.G. Hu and M.A. Dokainish, Damped vibrations of laminated composite plates - modeling and finite element analysis, Finite Elements in Analysis and Design 25 (1993), 103-124.

[7] S.J. Hwang and R.F. Gibson, The use of strain energy-based finite element techniques in the analysis of various aspects of damping of composite materials and structures, J. Composite Materials 26(17) (1992), 2585-2605.

[8] S.J. Hwang, R.F. Gibson and J. Singh, Decomposition of coupling effects on damping of laminated composites under flexural vibration, Composites Science and Technology 43 (1992), 159-169.

[9] T. Kant, J.H. Varaiya and C.P. Arora, Finite element transient analysis of composite and sandwich plates based on a refined theory and implicit time integration schemes, Computers \& Structures 36(3) (1990), 401-420.

[10] D.X. Lin, R.G. Ni and R.D. Adams, Prediction and measurement of the vibrational damping parameters of carbon and glass fiber reinforced plastic plates, J. Composite Materials 18 (1984).

[11] D.J. Mead and S. Markus, Coupled flexural longitudinal and shear wave motion in two and three-layered damped beams, J. Sound and Vibration 99(4) (1985), 501-519.

[12] D.R.J. Owen and Z.H. Li, A refined analysis of laminated plates by finite element displacement methods II - vibration and stability, Computers \& Structures 26(6) (1987), 915-923.

[13] T. Pervez and N. Zabaras, Transient dynamic and damping analysis of laminated anisotropic plates using a refined plate theory, Int. J. for Numerical Methods in Engineering 33 (1992), 1059-1080.

[14] D.A. Saravanos and C.C. Chamis, Computational simulation of damping in composite structures, J. Reinforced Plastics and Composites 10(3) (1991), 256-278.

[15] S. Shakhesi, Modèles de prédiction de l'amortissement des structures composites multicouches: approches numérique et expérimentale, optimisation. Thèse, Institut National Polytechnique de Grenoble, 1997.

[16] K.R. Sivadas and N. Ganesan, Effect of coupling between symmetric and antisymmetric modes in composite thick shells, $F i$ nite Elements in Analysis and Design 11 (1992), 9-18.

[17] C.T. Sun and H. Chin, On large deflection effects in unsymmetric cross-ply composite laminates, J. Composite Materials 22 (1988), 1045-1059.

[18] P. Trompette and R. Gaertner, Dynamic behavior of composite layered beams by the finite element method, The Shock and Vibration Bulletin (1983), 151-157. 

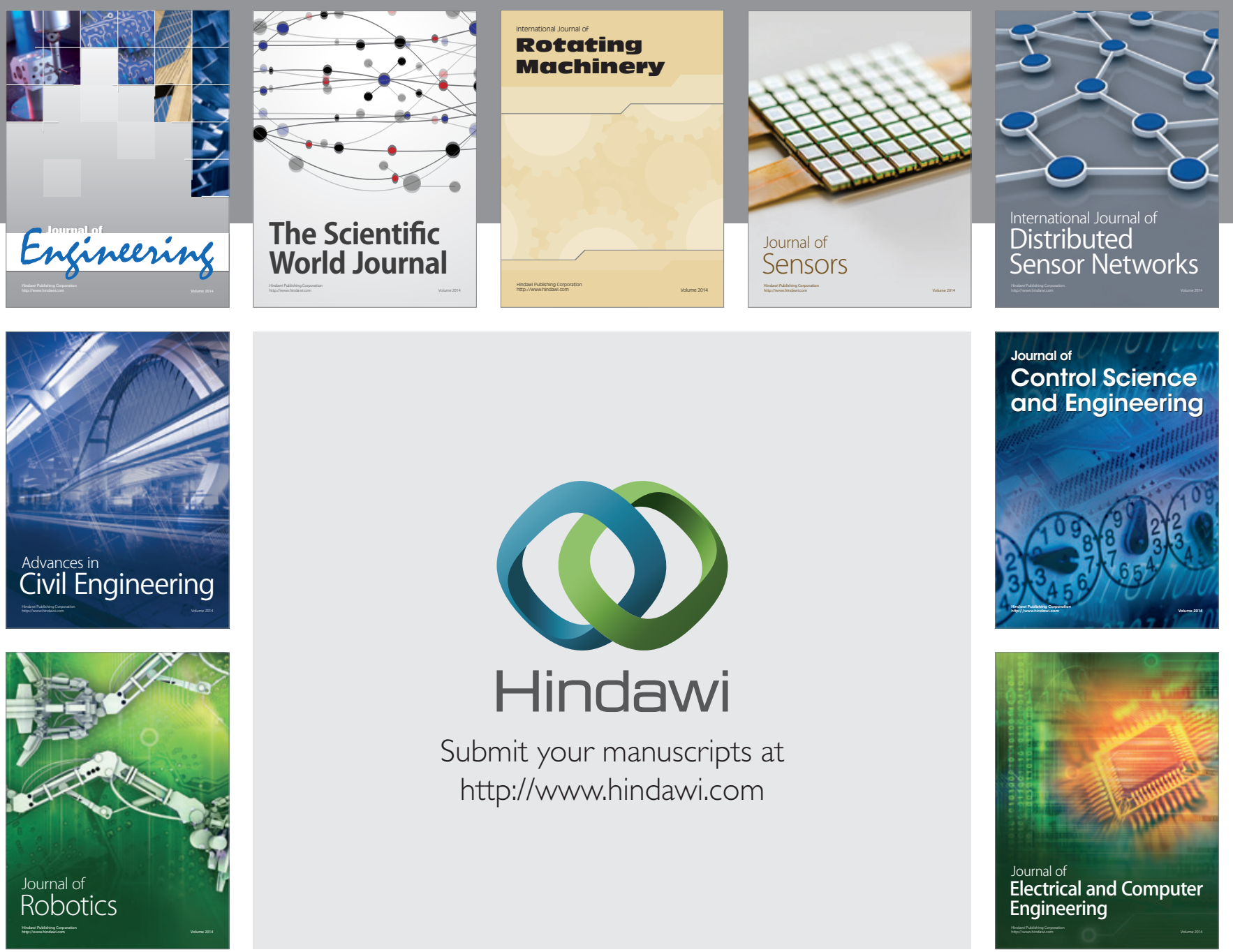

Submit your manuscripts at

http://www.hindawi.com
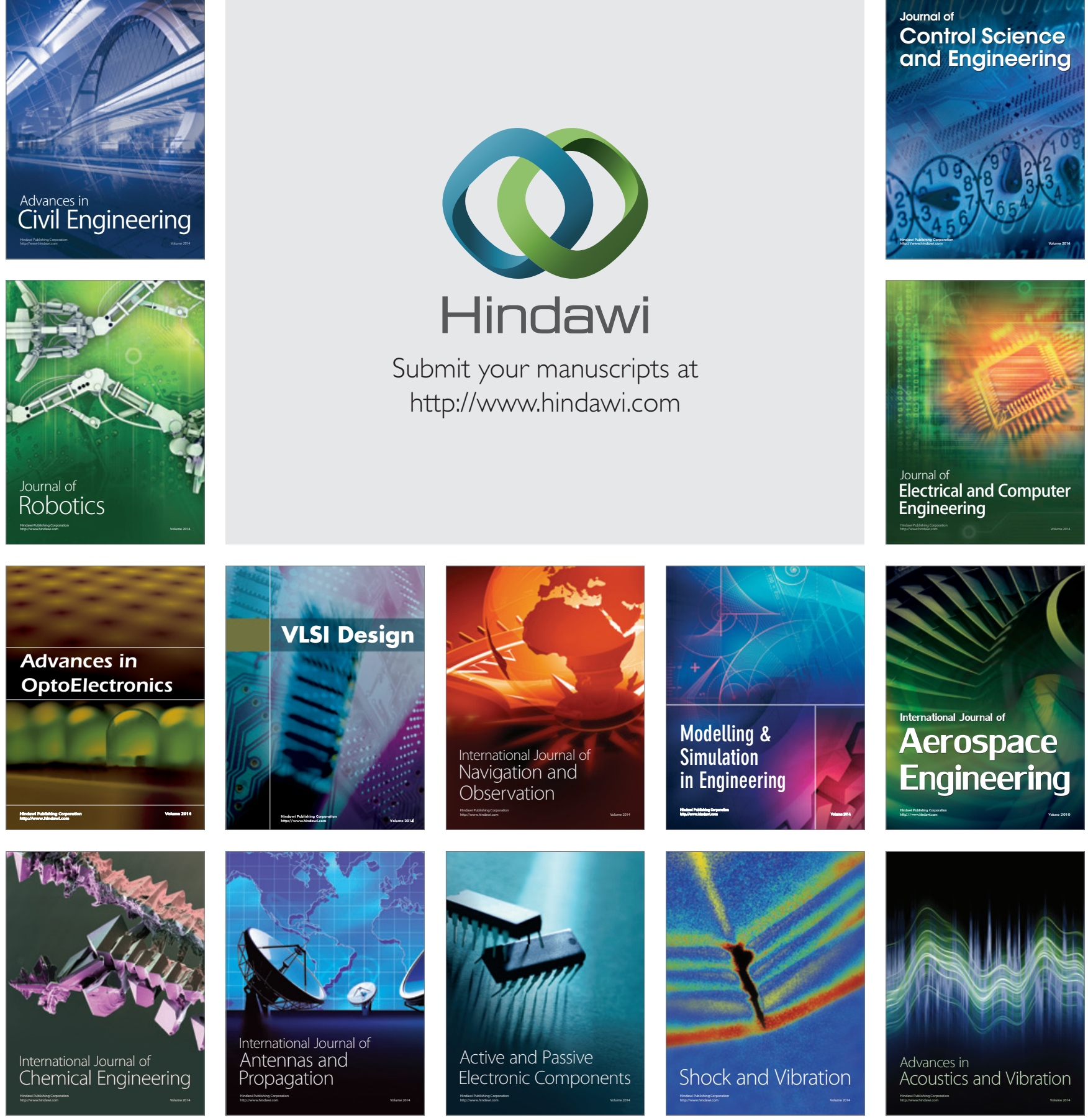